Disclosure of Interest: None declared

DOI: 10.1136/annrheumdis-2018-eular.1213

\section{THU0089 IL-17A+GM-CSF+ NEUTROPHILS ARE THE MAJOR INFILTRATING CELLS IN INTERSTITIAL LUNG DISEASE IN AN AUTOIMMUNE ARTHRITIS MODEL}

O.C. Kwon ${ }^{1}$, E.-J. Lee ${ }^{1}$, E.-J. Chang ${ }^{2}$, W.J. Seo ${ }^{3}$, J.S. Oh${ }^{4}$, S. Hong ${ }^{1}$, C.-K. Lee ${ }^{1}$, B. Yoo ${ }^{1}$, Y.-G. Kim ${ }^{1} .{ }^{1}$ Division of Rheumatology, Department of Medicine, University of Ulsan, College of Medicine, Asan Medical Center, ${ }^{2}$ Department of Biomedical Science, University of Ulsan, College of Medicine, Asan Medical Center, ${ }^{3}$ Division of Rheumatology, Department of Medicine, Seoul Veterans Hospital; ${ }^{4}$ Clinical Research Center, University of Ulsan College of Medicine, Asan Medical Center, Seoul, Korea, Republic of Ireland

Background: Interstitial lung disease (ILD) is a common extra-articular manifestation of rheumatoid arthritis (RA). Discrepancy in the effect of biologic agents on synovial and lung inflammation exists, indicating that the nature of inflammation in the synovium and lung may be different in RA

Objectives: To gain a better understanding of the pathogenesis of rheumatoid arthritis-associated interstitial lung disease (ILD), we sought to identify the characteristics of lung-infiltrating cells in SKG mice with ILD.

Methods: We injected curdlan in SKG mice at 8 weeks of age, and identified the presence of ILD by PET-MRI at 20 weeks post-injection and histological analysis at 22 weeks post-injection. Lung-infiltrating cells were examined by flow cytometry. Analysis of serum cytokines by the Luminex multiplex cytokine assay was performed at 14 and 22 weeks post-injection, and cytokine profiles before and afte the development of ILD were compared. Opal multiplexed immunofluorescent staining of lung tissue was also performed.

Results: At 20 weeks post-injection, curdlan-treated SKG mice developed not only arthritis but also lung inflammation combined with fibrosis, which was identified by PET-MRI and histological analysis. The majority of inflammatory cells that accumulated in the lungs of curdlan-treated SKG mice were CD11 ${ }^{+} \mathrm{Gr} 1^{+}$neutrophils, which co-express IL-17A and GM-CSF, rather than TNF- $\alpha$. Compared with 14 weeks post-injection, serum levels of GM-CSF, MCP1, IL-17A, IL-23, TSLP, and IL-7R $\alpha$ had increased at 22 weeks post-injection, whereas those of IFN- $\gamma$, IL22, IL-6, and TNF- $\alpha$ remained unchanged. Furthermore, IL-23, CXCL5, IL-17A, and GM-CSF, but not TNF- $\alpha$, were observed in immunofluorescent-stained lung tissue.

Conclusions: We found that $\mathrm{IL}-17 \mathrm{~A}^{+} \mathrm{GM}-\mathrm{CSF}^{+}$neutrophils represented the major inflammatory cells in the lungs of curdlan-treated SKG mice. In addition, GM-CSF and IL-17A appear to play a more important role than TNF- $\alpha$ in ILD development.

Acknowledgements: None.

Disclosure of Interest: None declared

DOI: 10.1136/annrheumdis-2018-eular.3399

\section{THU0090 PREVALENCE AND CLINICAL PHENOTYPE OF ANTI- CARBAMYLATED PROTEIN ANTIBODIES IN PALINDROMIC RHEUMATISM}

R. Castellanos-Moreira ${ }^{1}$, S. Cabrera-Villalba ${ }^{1}$, M. José Gomara ${ }^{2}$, V. Ruiz-Esquide ${ }^{1}$ G. Salvador ${ }^{3}$, S.D.C. Rodriguez-Garcia ${ }^{1}$, O. Camacho ${ }^{1}$, J. Ramírez $^{1}, \mathrm{M}$ V. Hernández ${ }^{1}$, A. Cuervo ${ }^{1}$, J.A. Gómez-Puerta ${ }^{1}$, J.D. Cañete ${ }^{1}$, I. Haro ${ }^{2}$ R. Sanmarti ${ }^{1} .{ }^{1}$ Rheumatology, Hospital Clinic de Barcelona; ${ }^{2}$ Unit of Synthesis and Biomedical Applications of Peptides, IQAC-CSIC; ${ }^{3}$ Rheumatology, Hospital Universitario Mutua Terrassa, barcelona, Spain

Background: Anti-carbamylated protein antibodies (anti-CarP) are a newly-identified antibody family present in the sera of rheumatoid arthritis (RA) patients whose specificity is close to that of ACPA but whose sensitivity is lower. Palindromic rheumatism (PR) is an intermittent form of arthritis that may progress to RA. Patients with PR may present the characteristic autoantibody profile seen in $R A$, even in the absence of evolution to RA. There are no studies on the presence of anti-CarP antibodies in PR.

Objectives: To analyse the prevalence of anti-CarP in patients with $\mathrm{PR}$ and evaluate their clinical phenotype and relationship with ACPA

Methods: Patients diagnosed with PR attended by the Arthritis Unit, Rheumatology Service, Hospital Clínic of Barcelona were included according to the criteria of Guerne et al. Only patients considered as pure PR (patients withou evidence of RA or other chronic rheumatic disease at the time of the first determination of anti-CarP) were analysed. An established RA control group was included. Anti-CarP were analysed by a home-made ELISA using a fibrin/ chimeric filaggrin homocitrulinated peptide as antigen. $\lg G \lg A$ and $\lg M$ isotypes were measured. ACPA were analysed with a commercial CCP2 test. The clinical and therapeutic characteristics were compared according to anti-
CarP positivity/negativity and to the clinical evolution, including the progression to RA during the follow-up.

Results: Anti-CarP were analysed in 54 patients with pure PR and 53 RA patients. Anti-CarP were detected in $16.7 \%$ and $37.7 \%$ (p: NS) of patients with PR and RA, respectively. All PR patients positive for anti-CarP were ACPA positive and had higher ACPA titers. IgG was the predominant isotype $(100 \%)$, only one patient presented $\lg \mathrm{A}(11.1 \%)$ and none $\lg \mathrm{M}$. In the RA group, the percentages of $\lg$, $\lg A$ and $\lg M$ were $55 \%, 50 \%$ and $40 \%$, respectively, in anti-CarP positive patients. More anti-CarP positive patients developed RA (33.3\% vs. 13.6\%), although the difference was not significant, during a mean follow-up of $3.8 \pm 1.1$ years. Remission at the last follow-up visit was more frequent in patients who were anti-CarP negative ( $22 \%$ vs. $60.5 \% \mathrm{p}<0.05)$. Anti-CarP positive patients were receiving DMARD more frequently than anti-CarP negative patients $(100 \%$ vs. $60 \%$ p $<0.05$ )

Conclusions: This is the first study to demonstrate the presence of anti-CarP in patients with pure PR. Anti-CarP was associated with ACPA positivity and higher ACPA titers. Anti-CarP isotypes differed between PR and RA, with lower isotype use in PR. Anti-CarP positivity in PR patients could indicate a more refractory disease, with lower rates of remission and greater predisposition to evolve to RA.

Acknowledgements: This study was supported by two awards granted by Hos pital Clínic de Barcelona, Research, Innovation and Education Department (Sonia Cabrera-Villalba, Premi Fi de residencia: Emili Letang 052012; Raul CastellanosMoreira, Premi Fi de residencia: Emili Letang 379_33_PFR_250760_2017).

Disclosure of Interest: None declared DOI: 10.1136/annrheumdis-2018-eular.4964

\section{THU0091 TIE2 INDUCES AN INFLAMMATORY PHENOTYPE IN RHEUMATOID ARTHRITIS AND PSORIATIC ARTHRITIS PATIENTS DRIVEN BY ANGIOPOIETIN-2}

S. García Pérez ${ }^{1}$, P.A. Kabala ${ }^{1}$, B. Malvar Fernández ${ }^{1}$, M.W. Tang ${ }^{2}$, S A. Hartgring ${ }^{1}$, C. Conde ${ }^{3}$, M. Sleeman ${ }^{4}$, D.L. Baeten ${ }^{2}$, P.P. Tak ${ }^{5}$, J. Connor ${ }^{6}$, K. A. Reedquist ${ }^{1}$, T.R. Radstake ${ }^{1} .{ }^{1}$ Laboratory for Translational Immunology (LTI) and Department of Rheumatology and Clinical Immunology, University Medical Center Utrecht, Utrecht, ${ }^{2}$ Department of Clinical Immunology and Rheumatology, Academic Medical Center, University of Amsterdam, Amsterdam, Netherlands; ${ }^{3}$ Laboratorio de Investigación 8 y Servicio de Reumatología, Instituto de Investigación Sanitaria (IDIS), Hospital Clínico Universitario de Santiago de Compostela (CHUS), Santiago de Compostela, Spain; ${ }^{4}$ Regeneron Pharmaceuticals, Inc., Tarrytown, New York, USA; ${ }^{5}$ GlaxoSmithKline, Stevenage, UK; ${ }^{6}$ Medlmmune LCC, Maryland, USA

Background: Several studies have shown that angiopoietin signalling to Tie2 may play a role in the initiation and perpetuation of disease rheumatoid arthritis (RA) and psoriatic arthritis (PsA). However, the cell type(s) involved in this process, as well the specific role of Tie2 signalling in the synovium of arthritis patients remain unclear.

Objectives: To examine the role Tie2 signalling in macrophages and fibroblastlike synoviocytes (FLS) within the context of the synovial microenvironment of arthritic patients.

Methods: Peripheral blood (PB) monocytes from healthy donors (HD) were differentiated with synovial fluid (SF) of RA and PsA patients. PB and SF monocytes from RA and PsA patients were differentiated into pro-inflammatory macrophages with IFN- $\gamma$. Tie2 expression was analysed by flow cytometry and quantitative PCR. Macrophages, RA FLS and synovial tissue explants were stimulated with Ang-1 or Ang-2 $(200 \mathrm{ng} / \mathrm{ml})$ alone or in combination with TNF $(10 \mathrm{ng} / \mathrm{ml})$ for 4 hour or 24 hour. mRNA and protein expression of inflammatory mediators was analysed by quantitative PCR and ELISA and luminex, respectively. Arthritis was induced in wild type (WT) and Tie2 over-expressing (Tie2-TG) mice by intraperitoneal injection of $100 \mathrm{ml}$ of $\mathrm{K} / \mathrm{BxN}$ serum on day 0 and day 2 . Mice were sacrificed on day 14 after serum transfer.

Results: Tie2 expression was observed IFN- $\gamma$-differentiated macrophages, from RA and PsA patients, as well as HD macrophages differentiated with RA and PsA SF. In all cases, both Ang-1 and Ang-2 stimulation significantly enhanced TNFinduced expression of pro-inflammatory cytokines (IL-6, IL-12B) and chemokines (IL-8, CCL-3 and CXCL-6). Tie2 activation also enhanced TNF-mediated production of these inflammatory mediators in RA FLS. The clinical severity of serum induced arthritis was significantly higher in Tie2-TG mice compared to WT mice associated with enhanced synovial expression of IL-6, IL12B, iNOS, CCL-2 and CXCL-10.

Finally, we found that Ang-2, and to a lower extent Ang-1, induced the production of IL-6, IL-12B, IL-8, and CCL-3 in the synovial tissue explants of RA and PSA patients. Importantly, Ang-2 blockade with a specific neutralising anti-Ang2 antibody suppressed the production of IL- 6 and IL-8 in synovial tissue of RA patients. Conclusions: These results suggest that Tie2 signalling, even within the com plex microenvironment of affected synovial tissue, has an important pro- 